\title{
Simultaneous Determination of Ginkgo \\ Flavonoids and Terpenoids in Plasma: Ammonium Formate in LC Mobile Phase Enhancing Electrospray Ionization Efficiency and Capacity
}

\author{
Yang Zhao, Yan Sun, and Chuan Li \\ Shanghai Institute of Materia Medica, Chinese Academy of Sciences, Shanghai, China
}

Extracts from Ginkgo biloba leaves confer their therapeutic effects through the synergistic actions of flavonoid and terpenoid components. We herein describe the development of an LC-MS/MS-based method for simultaneous determination of flavonoids (quercetin, kaempferol, and isorhamnetin) and terpenoids (bilobalide, ginkgolides A, B, C, and J) in acid-hydrolyzed plasma by circumventing cross-interference between the flavonoids and terpenoids identified. Notably, inclusion of ammonium formate $(0.2 \mathrm{mM})$ in the mobile phase generated beneficial LC-electrolyte effects, including increased ESI efficiency and capacity, with the result that the newly developed procedure exhibits the highest analytical performance reported to date for ginkgo-associated studies. The method yields high sensitivity, negligible matrix interference and cross-interference, wide linear dynamic ranges, high sample throughput, and quite small initial sample size. The assay utility to dog pharmacokinetic measurements of commercial ginkgo products yielded the most comprehensive data on systemic exposure to the ginkgo compounds to date. The newly developed multi-analyte procedure should be widely useful. (J Am Soc Mass Spectrom 2008, 19, 445-449) (c) 2008 American Society for Mass Spectrometry

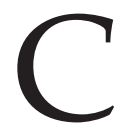
linical studies have suggested that the consumption of extracts of Ginkgo biloba leaves (family Ginkgoaceae) can enhance memory and alleviate symptoms of Alzheimer's disease. The positive effects of ginkgo extracts are presumed to result from the synergistic action of two distinct classes of constituents, the flavonoids (glycosides of quercetin, kaempferol, and isorhamnetin) and terpenoids (bilobalide, ginkgolides A, B, C, and J) [1].

Many earlier pharmacokinetic measurements involved the measurement of only plasma ginkgo terpenoids $[2,3]$. Recently, we developed a sensitive liquid chromatography-tandem mass spectrometry (LC-MS/MS)-based method for quantification of ginkgo flavonoids in acidhydrolyzed plasma for routine PK measurements of ginkgo-based products [4]. However, it would be highly beneficial to develop a rapid method capable of analyzing both classes of the ginkgo compounds in parallel from relatively small amounts of biological samples. Ding et al. developed a capillary LC-MS

Address reprint requests to Dr. C. Li, Shanghai Center for DMPK Research, Shanghai Institute of Materia Medica, SIBS, Chinese Academy of Sciences, 555 Zuchongzhi Road, Zhangjiang Hi-Tech Park, Shanghai 201203, China. E-mail: chli@mail.shcnc.ac.cn method for simultaneous quantification of ginkgo flavonoids and terpenoids excreted in human urine [5], but its chromatographic separation required $140 \mathrm{~min} /$ sample. PK studies of botanical drugs or nutritional products call for high-throughput and sensitive bioanalytical assays.

In this study, we report development of a new LC-MS/MS-based method for simultaneous determination of 8 ginkgo flavonoids and terpenoids in plasma, which has higher overall analytical performance than previously described methods with respect to sensitivity, initial plasma sample size, and speed.

\section{Experimental}

\section{Reference Standards}

Standards of bilobalide (BLL; MW, 326), ginkgolides A (GLA; 408), B (GLB; 424), C (GLC; 440), quercetin (QCT; 302), kaempferol (KMF; 286), and isorhamnetin (ISR; 316) were obtained from the National Institute for the Control of Pharmaceutical and Biological Products (Beijing, China). Reference standard of ginkgolide J (GLJ; 424) was purchased from Tauto 
Biotech (Shanghai, China). The purity of these standards was $\geq 99.0 \%$.

\section{Sample Clean-up}

Plasma samples (20 $\mu \mathrm{L}$ each) were treated with $10 \mathrm{mM}$ ascorbic acid before storage at $-70{ }^{\circ} \mathrm{C}$. For clean-up, the thawed plasma samples were treated with $20 \mu \mathrm{L}$ of $4 \mathrm{M}$ hydrochloric acid at $80^{\circ} \mathrm{C}$ for $30 \mathrm{~min}$, and then extracted with $600 \mu \mathrm{L}$ of ethyl acetate and centrifuged. The upper supernatant $(500 \mu \mathrm{L})$ was dried under a stream of $\mathrm{N}_{2}$. The residue was reconstituted in $40 \mu \mathrm{L}$ of acetonitrile, centrifuged, and $5 \mu \mathrm{L}$ of the resulting supernatant were applied to LC-MS/MS analysis.

\section{LC-MS/MS Conditions}

The LC-MS/MS system consisted of a Thermo Fisher TSQ Quantum mass spectrometer (San Jose, CA) interfaced by an electrospray ionization (ESI) probe with an Agilent 1100 LC system (Waldbronn, Germany). Chromatographic separations were achieved using a Phenomenex Gemini $5 \mu \mathrm{m} \mathrm{C18}$ column $(50 \times 2.0 \mathrm{~mm}$ i.d.; Torrance, CA). The mobile phases (delivered at 0.3 $\mathrm{mL} / \mathrm{min}$ ) consisted of $\mathrm{CH}_{3} \mathrm{CN} / \mathrm{H}_{2} \mathrm{O}$ (5:495, vol/vol, containing $0.2 \mathrm{mM} \mathrm{HCOONH}_{4}$ ) for $A$ and $\mathrm{CH}_{3} \mathrm{CN} / \mathrm{H}_{2} \mathrm{O}$ (450:50, vol/vol, containing $0.2 \mathrm{mM} \mathrm{HCOONH}_{4}$ ) for $B$. A binary pulse gradient elution was performed, which consisted of a 0.1-min initial increase of $B$ from 15 to $70 \%$, followed by maintaining $B$ at $70 \%$ from 0.1 to 1.3 $\mathrm{min}$. At $1.4 \mathrm{~min}, B$ was quickly returned to $15 \%$ and maintained until $5 \mathrm{~min}$.

The MS/MS parameters in the negative-ion ESI mode were tuned to maximize generation of deprotonated molecules for the analytes, except for GLA and GLJ, using the formate adduct, and to produce characteristic product ions for each analyte. The precursor-toproduct ion pairs used for selected reaction monitoring of QCT, KMF, ISR, BLL, GLA, GLB, GLC, and GLJ were $\mathrm{m} / \mathrm{z} 301 \rightarrow 151$ (the optimal collision energy, $29 \mathrm{~V}$ ), $285 \rightarrow 187(37 \mathrm{~V}), 315 \rightarrow 300(28 \mathrm{~V}), 325 \rightarrow 163(27 \mathrm{~V})$, $453 \rightarrow 351(27 \mathrm{~V}), 423 \rightarrow 367(22 \mathrm{~V}), 439 \rightarrow 383(21 \mathrm{~V})$, and $469 \rightarrow 423(15 \mathrm{~V})$, respectively, with a scan time of $0.1 \mathrm{~s}$ for each ion pair. Only LC eluent flow over a period of 2.7 to $4.6 \mathrm{~min}$ was introduced to the mass spectrometer for data acquisition.

Standard curves were constructed within the plasma concentration range 0.128 to $2000 \mathrm{ng} / \mathrm{mL}$ using weighted $\left(1 / X^{2}\right)$ linear regressions of the peak areas of the analyte $(Y)$ against the corresponding nominal plasma concentrations of the analyte $(X, \mathrm{ng} / \mathrm{mL})$.

\section{Assessment of Matrix Effects and Method Validation}

Matrix effect window, as well as absolute and relative matrix effects, were examined as described in our earlier publication $[4,6]$.
Assay validation was conducted to demonstrate that the performance characteristics of the newly developed method were suitable and reliable for the intended applications. The criteria used to validate the method for quantification of multiple analytes from plasma should not be compromised for each analyte in accuracy, precision, selectivity, sensitivity, reproducibility, and stability. Each analyte was tested to ensure no cross-interference from the other analytes.

\section{Results and Discussion}

\section{Sensitive ESI-MS/MS of All the Ginkgo Analytes}

In this study, we aimed to develop a single LC-MS/MSbased method for simultaneous analysis of both flavonoids and terpenoids in plasma. Previously, we found that negative-ion ESI provided high ionization efficiency for the ginkgo flavonoids QCT, KMF, and ISR [4], and that this efficiency could be enhanced by the use of a mobile phase modified with $0.01 \%$ o $(\mathrm{mL} / \mathrm{mL})$ $\mathrm{HCOOH}$ (the so called "LC-electrolyte effect" [6]). Thus, we assessed the inherent suitability of the ginkgo terpenoids for analysis in negative-ion ESI mode. The peaks attributable to the deprotonated molecule $[\mathrm{M}-\mathrm{H}]^{-}$and the formate adduct $[\mathrm{M}+\mathrm{HCOO}]^{-}$were the major peaks in all spectra of the tested terpenoids, while the small ion peaks $[2 \mathrm{M}-\mathrm{H}]^{-}$were also seen. BLL was poorly ionized by the positive ion ESI. Due to poor sensitivity, APCI was not considered in this study either. Collectively, the negative-ion ESI was the most suitable for the study purpose.

ESI signal intensity of a given analyte is a function of the percentage of electrolytic content in mobile phase $[6,7]$. We found that the highest ESI response was achieved for all analytes when the mobile phase included $0.2 \mathrm{mM} \mathrm{HCOONH}_{4}$. Despite significant structural diversity among the flavonoids and the terpenoids, there was a uniform dependence of signal intensity on $\mathrm{HCOONH}_{4}$ concentrations (0-25 mM). Although the signal intensity at $m / z[\mathrm{M}-\mathrm{H}]^{-}$and $[\mathrm{M}+\mathrm{HCOO}]^{-}$of the terpenoids changed in almost the same profile shapes as the electrolyte concentration increased, these profiles were seen at different response levels, which were analyte-dependent. This observation suggested that the LC-electrolyte effect in signal enhancement was nonselective for different ions generated from the analyte. Sensitive and specific MS/MS detection was achieved by using the precursor-to-product ion pairs given in the Experimental section.

\section{Improvement of Upper Limits of Quantification}

In this study, we investigated whether adding the electrolyte $\mathrm{HCOONH}_{4}$ to the LC mobile phase could improve the upper limits of ESI-quantification. Because greater deviations present at higher concentrations tend to weight the calibration curve more than smaller deviations associated with lower concentrations in least 
square linear regression [8], we used plasma calibration standards of low concentrations to generate calibration curves for the analytes, and the resulting linear regression equations were used to calculate the theoretical detection responses of each analyte at higher concentrations. Herein, the low concentrations included 0.640, $3.20,16.0$, and $80.0 \mathrm{ng} / \mathrm{mL}$ and the plasma calibration standards contained equal concentrations of the ginkgo analytes, whereas the tested higher concentration were 400, 2000, and $10000 \mathrm{ng} / \mathrm{mL}$. Then, the measured higher concentrations were compared with the corresponding calculated values. The criteria used to determine the upper limits of linear dynamic range were the mean measured response $(n=3)$ showing $\geq 85 \%$ accuracy compared with the calculated value and $\leq 15 \%$ precision.

Using the preceding method, we found that the linear dynamic ranges of the analytes never exceeded 80 or $400 \mathrm{ng} / \mathrm{mL}$ with electrolyte-free mobile phase. In contrast, when mobile phase was modified with $\mathrm{HCOONH}_{4}$, the upper limits extended to a higher concentration (Table 1), except for GLC but showing better analytical accuracy at $400 \mathrm{ng} / \mathrm{mL}$ with $\mathrm{HCOONH}_{4}$-mobile phases than electrolyte-free one. As to the effect of $\mathrm{HCOONH}_{4}$ concentration, greater extension of the upper limits were achieved for the flavonoids using $0.2 \mathrm{mM} \mathrm{HCOONH}_{4}$, and for BLL and GLJ using $5 \mathrm{mM} \mathrm{HCOONH}_{4}$, whereas compara- ble results were obtained for the other ginkgolides. Similar results on the effect of $\mathrm{HCOONH}_{4}$ were obtained using matrix-component free calibration standards prepared in $\mathrm{CH}_{3} \mathrm{CN}$ (data not shown).

Although others have explored many avenues for improving ESI efficiency, the efforts have failed to extend the upper limit of quantification [9]. Our study yields the important finding that modification of the mobile phase with $\mathrm{HCOONH}_{4}$ appears to extend the upper limit of ESI quantification, which is advantageous for quantitative analysis in PK studies.

\section{Reduction of Matrix Effects and Chromatographic Run Time}

When using electrolyte-free mobile phase, both the tested flavonoids and terpenoids showed the same initial ion enhancement ( $\sim 3$ - to 5-fold) after injection of prepared blank canine plasma samples, and it took $\sim 6 \mathrm{~min}$ for the system to regain its presample injection intensity. These adverse matrix effects were significantly minimized by using a mobile phase modified with $\mathrm{HCOONH}_{4}(0.2 \mathrm{mM})$, as demonstrated by the observation of matrix effect windows for the analytes measured at $[\mathrm{M}-\mathrm{H}]^{-}$or $[\mathrm{M}+$ $\mathrm{HCOO}^{-}$reduced to $<3 \mathrm{~min}$. This favorable effect of $\mathrm{HCOONH}_{4}$ enabled us to develop a rapid single LCMS/MS method for simultaneous determination of the

Table 1. Effect of concentration of $\mathrm{HCOONH}_{4}$ in LC mobile phases on upper limits of quantification of the Ginkgo analytes

\begin{tabular}{|c|c|c|c|}
\hline \multirow[b]{2}{*}{ Analyte } & \multicolumn{3}{|c|}{$\begin{array}{c}\text { Regression equation }^{\mathrm{a}} \\
\text { [Tested concentration }(\mathrm{ng} / \mathrm{mL})] \text { Accuracy }(\%) / \text { Precision (\%) }\end{array}$} \\
\hline & $0 \mathrm{mM} \mathrm{HCOONH}_{4}$ & $0.2 \mathrm{mM} \mathrm{HCOONH}_{4}$ & $5 \mathrm{mM} \mathrm{HCOONH}_{4}$ \\
\hline QCT & $\begin{aligned} Y= & 4278 X-1320 \\
& {[80] 100 / 2.0 } \\
& {[400] 80 / 1.0 }\end{aligned}$ & $\begin{array}{c}Y=12814 X+7682 \\
{[10000] 87 / 3.7}\end{array}$ & $\begin{array}{r}Y=8316 X-480 \\
{[400] 85 / 6.4} \\
{[2000] 50 / 4.9}\end{array}$ \\
\hline KMF & $\begin{array}{r}Y=568 X+210 \\
{[80] 100 / 5.7} \\
{[400] 78 / 3.9}\end{array}$ & $\begin{aligned} Y= & 2280 X+1208 \\
& {[400] 104 / 5.6 } \\
& {[2000] 80 / 5.5 }\end{aligned}$ & $\begin{array}{r}Y=780 X-1202 \\
{[400] 99 / 2.2} \\
{[2000] 68 / 0.21}\end{array}$ \\
\hline ISR & $\begin{aligned} Y= & 14680 X+24040 \\
& {[80] 100 / 6.7 } \\
& {[400] 65 / 2.9 }\end{aligned}$ & $\begin{array}{c}Y=71866 X+6844 \\
{[400] 101 / 4.6} \\
{[2000] 73 / 4.5}\end{array}$ & $\begin{array}{c}Y=28874 X+39192 \\
{[80] 100 / 4.0} \\
{[400] 79 / 4.0}\end{array}$ \\
\hline BLL & $\begin{array}{r}Y=5376 X+78 \\
{[400] 94 / 4.4} \\
{[2000] 63 / 2.4}\end{array}$ & $\begin{array}{c}Y=24746 X+706 \\
{[400] 99 / 1.3} \\
{[2000] 82 / 2.6}\end{array}$ & $\begin{array}{c}Y=5886 X+1722 \\
{[2000] 89 / 0.51} \\
{[10000] 70 / 0.73}\end{array}$ \\
\hline GLA & $\begin{aligned} Y= & 3934 X+2532 \\
& {[80] 100 / 2.4 } \\
& {[400] 80 / 0.87 }\end{aligned}$ & $\begin{array}{c}Y=25514 X+2134 \\
{[400] 92 / 0.85} \\
{[2000] 70 / 1.1}\end{array}$ & $\begin{aligned} Y= & 5854 X-594 \\
& {[400] 111 / 0.43 } \\
& {[2000] 80 / 0.18 }\end{aligned}$ \\
\hline GLB & $\begin{aligned} Y= & 6002 X+3568 \\
& {[80] 100 / 1.7 } \\
& {[400] 83 / 1.6 }\end{aligned}$ & $\begin{array}{c}Y=34128 X+7278 \\
{[400] 90 / 0.95} \\
{[2000] 71 / 0.24}\end{array}$ & $\begin{aligned} Y= & 7936 X+2960 \\
& {[400] 106 / 1.7 } \\
& {[2000] 79 / 0.60 }\end{aligned}$ \\
\hline GLC & $\begin{array}{c}Y=4826 X-530 \\
{[400] 88 / 2.3} \\
{[2000] 53 / 0.82}\end{array}$ & $\begin{aligned} Y= & 16534 X-1614 \\
& {[400] 101 / 2.3 } \\
& {[2000] 76 / 0.43 }\end{aligned}$ & $\begin{aligned} Y= & 4836 X-2348 \\
& {[400] 102 / 2.2 } \\
& {[2000] 79 / 0.61 }\end{aligned}$ \\
\hline GLJ & $\begin{array}{r}Y=4552 X-594 \\
{[400] 91 / 0.87} \\
{[2000] 54 / 1.1}\end{array}$ & $\begin{array}{c}Y=31208 X-6828 \\
{[400] 97 / 0.81} \\
{[2000] 74 / 1.1}\end{array}$ & $\begin{array}{c}Y=7628 X-1242 \\
{[2000] 86 / 1.2} \\
{[10000] 79 / 1.4}\end{array}$ \\
\hline
\end{tabular}

${ }^{a} Y$ : analyte peak area; $X$ concentration of analyte in the injected solution in $\mathrm{ng} / \mathrm{mL}$. The study calibration curves were prepared by using post-extracted blank plasma samples spiked with a mixture of the analytes at equal concentrations $(0.640-80.0 \mathrm{ng} / \mathrm{mL})$, and the sample injection volume was $5 \mu \mathrm{L}$. 
eight analytes in plasma with a chromatographic run time of only $5 \mathrm{~min}$. The retention times of GLC, GLJ, BLL, GLA, GLB, QCT, KMF, and ISR were 3.3, 3.3, 3.5, 3.7, 3.7, 3.8, 4.1, and $4.1 \mathrm{~min}$, respectively.

Ionization efficiency can be defined as the ratio between the number of analyte ions recorded versus the number of analyte molecules sprayed. It represents a combination of analyte charging, droplet evaporation, and ion transmission efficiencies in the vacuum system. The results from our current and earlier studies $[4,6,7]$ indicate that the electrolyte modification of mobile phase can significantly improve the ESI efficiency, resulting in enhanced analyte responses. For quantification purposes, the magnitude of ionization efficiency should be maintained constantly throughout an analyte concentration range; the ability to hold the ionization efficiency is called the "ionization capacity". In this study, the use of an $\mathrm{HCOONH}_{4}$-modified mobile phase yielded the enlarged linear dynamic range and minimized the matrix effect window, suggesting that the electrolyte increased the ESI capacity for the tested compounds under the "crowded" situations occurring in the ESI droplets. Modifying the mobile phase with $\mathrm{HCOONH}_{4}$ enhanced solution conductivity and may reduce the initial droplet size [10]. Due to enhancement in overall droplet surface-to-volume ratio, the ESI efficiency can be better maintained in "crowded" situations.

\section{Demonstration and Circumvention of Analyte Cross-Interferences}

In this study, we found significant cross-interference between the ginkgo flavonoids and terpenoids (Table 2). The plasma calibration standards containing only the flavonoids or the terpenoids showed good linear relationships between the plasma concentration and the ESIMS/MS response, as indicated by good correlation coefficients $(r \geq 0.995)$ and good precisions (RSD: $4 \%$ to $13 \%)$ for all the analytes. However, when the calibration standards containing both the classes of analytes were examined, the linear relationships became poor, as evidenced by $r=0.420-0.782$ for the flavonoids and $0.984-0.987$ for the terpenoids, and RSD $=14 \%$ to $114 \%$ and $4 \%$ to $22 \%$, respectively. In addition, the slopes of the regression equations were also significantly altered. The interference was mainly due to the coexistence of the flavonoids and terpenoids, as confirmed by examining matrix component-free calibration standards. Thorough LC separation of all the eight analytes failed to overcome the cross-interference problem. Instead, we found that this problem could be counteracted by adding ascorbic acid to the plasma samples before sample clean-up and by reconstituting the extract residues in absolute $\mathrm{CH}_{3} \mathrm{CN}$ before LC-MS/MS (Table 2).

\section{Assay Validation and Applicability Demonstration}

The ginkgo analytes were stable under conditions mimicking those experienced by real samples. No analyte cross-interference was found and the method specificity was demonstrated by no co-eluted endogenous compounds at peaks corresponding to the analytes. The extraction efficiencies excluding matrix effects were $42 \%$ to $65 \%$ for the analytes. The absolute and relative matrix effects from the plasma samples on analyte ionization were $-14.9 \%$ to $7.0 \%$ and $<13.8 \%$, respectively. The matrix-based calibration curves were linear with corre-

Table 2. Cross-interference between the Ginkgo flavonoids and the Ginkgo terpenoids

Regression equation ${ }^{a}$

(Correlation coefficient; RSD range)

\begin{tabular}{|c|c|c|c|}
\hline Analyte & $\mathrm{F}$ or $\mathrm{T}$ alone $\mathrm{e}^{\mathrm{b}}$ & $\mathrm{F}+\mathrm{T}^{\mathrm{c}}$ & $(F+T)+A^{d}$ \\
\hline QCT & $\begin{array}{c}Y=4227 X-411 \\
(0.997 ; 4 \%-10 \%)\end{array}$ & $\begin{array}{c}Y=413 X+20053 \\
(0.420 ; 14 \%-114 \%)\end{array}$ & $\begin{array}{c}Y=3971 X+2399 \\
(0.999 ; 3 \%-7 \%)\end{array}$ \\
\hline KMF & $\begin{array}{l}Y=611 X+31 \\
(0.999 ; 4 \%-8 \%)\end{array}$ & $\begin{array}{l}Y=240 X+389 \\
(0.782 ; 56 \%-102 \%)\end{array}$ & $\begin{array}{l}Y=598 X-106 \\
(0.999 ; 3-10 \%)\end{array}$ \\
\hline ISR & $\begin{array}{c}Y=18020 X+5144 \\
(0.999 ; 3 \%-6 \%)\end{array}$ & $\begin{array}{c}Y=4971 X+41597 \\
(0.653 ; 27 \%-112 \%)\end{array}$ & $\begin{array}{c}Y=17049 X+1264 \\
(0.999 ; 0 \%-4 \%)\end{array}$ \\
\hline BLL & $\begin{array}{c}Y=7637 X+474 \\
(0.999 ; 4 \%-11 \%)\end{array}$ & $\begin{array}{l}Y=5738 X+200 \\
(0.984 ; 4 \%-16 \%)\end{array}$ & $\begin{array}{c}Y=7339 X+228 \\
(0.999 ; 1 \%-5 \%)\end{array}$ \\
\hline GLA & $\begin{array}{c}Y=5889 X+1087 \\
(0.998 ; 5 \%-8 \%)\end{array}$ & $\begin{array}{l}Y=4913 X+245 \\
(0.986 ; 7 \%-18 \%)\end{array}$ & $\begin{array}{c}Y=5386 X+1302 \\
(0.999 ; 1 \%-6 \%)\end{array}$ \\
\hline GLB & $\begin{array}{r}Y=8794 X+323 \\
(0.998 ; 4 \%-11 \%)\end{array}$ & $\begin{array}{l}Y=7694 X+239 \\
(0.987 ; 4 \%-18 \%)\end{array}$ & $\begin{array}{c}Y=8558 X+1076 \\
(0.999 ; 1 \%-5 \%)\end{array}$ \\
\hline GLC & $\begin{array}{c}Y=4825 X-610 \\
(0.998 ; 4 \%-9 \%)\end{array}$ & $\begin{array}{c}Y=4302 X+502 \\
(0.985 ; 5 \%-21 \%)\end{array}$ & $\begin{array}{l}Y=4980 X-67 \\
\quad(0.999 ; 1 \%-12 \%)\end{array}$ \\
\hline GLJ & $\begin{array}{c}Y=8932 X+11787 \\
(0.995 ; 5 \%-13 \%)\end{array}$ & $\begin{array}{l}Y=6402 X+21612 \\
(0.984 ; 7 \%-22 \%)\end{array}$ & $\begin{array}{c}Y=8204 X+21950 \\
(0.999 ; 2 \%-6 \%)\end{array}$ \\
\hline
\end{tabular}

a $Y$ : analyte peak area; $X$ : concentration of analyte in the initial plasma sample in $\mathrm{ng} / \mathrm{mL} ; n=3$.

${ }^{\mathrm{b}} \mathrm{F}$ or $\mathrm{T}$ alone: either the 3 tested flavonoids or the 5 tested terpenoids contained in the plasma standards treated without ascorbic acid.

${ }^{c} \mathrm{~F}+\mathrm{T}$ : all the 8 analytes in the plasma standards treated without ascorbic acid.

$\mathrm{d}(\mathrm{F}+\mathrm{T})+\mathrm{A}$ : all the 8 analytes in the plasma standards treated with ascorbic acid $(10 \mathrm{mM})$ and then the EtOAc extract residues reconstituted in absolute $\mathrm{CH}_{3} \mathrm{CN}$. 


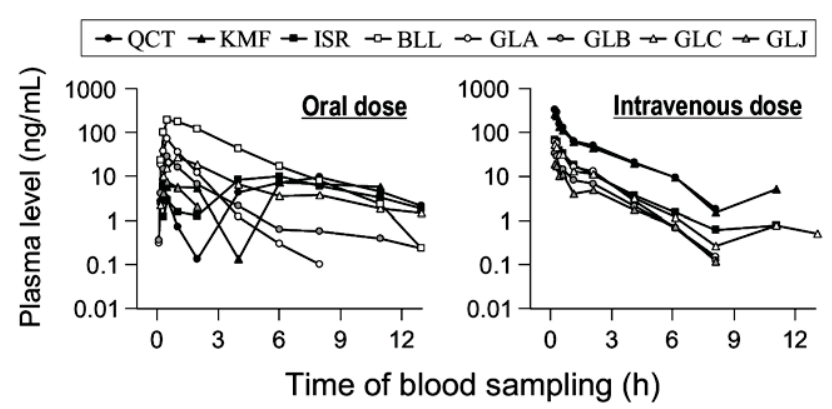

Figure 1. Plots of plasma concentrations of the tested ginkgo flavonoids and terpenoids against the time of blood sampling. A male beagle dog was given, on separate occasions, a single oral dose of 2 Ginaton film-coated tablets or a single intravenous dose of $5 \mathrm{~mL}$ of Ginaton injection solution. Serial blood samples $(0,5$, 10, 20, $30 \mathrm{~min}, 1,2,4,6,8,11$, and $13 \mathrm{~h}$ ) were collected in heparinized tubes and centrifuged to yield plasma samples.

lation coefficients of 0.999 . The within-run $(n=5)$ and between-run $(n=3)$ assay precisions for all analytes were $1.1 \%$ to $18.5 \%$ and $1.0 \%$ to $19.3 \%$, respectively. The corresponding accuracies were $82 \%$ to $115 \%$ and $97 \%$ to $120 \%$, respectively. The on-column lower limits of quantification were $0.32 \mathrm{pg}$, except for GLJ (1.6 pg).

To demonstrate the applicability of our new method, we used it to analyze plasma samples obtained from a dog PK study of two standardized ginkgo extract preparations. As shown in Figure 1, all of the tested analytes were detected in the canine plasma samples following oral or intravenous dosing. The plasma concentration-time data enabled us to calculate the PK parameters reflecting the systemic exposure, distribution, and elimination properties of the tested ginkgo compounds.

\section{Conclusions}

We herein developed the first LC-MS/MS-based procedure in the negative-ion ESI mode for simultaneous measurement of eight ginkgo flavonoids and terpenoids in plasma samples. A significant cross-interference was observed between the ginkgo flavonoids and terpenoids that lies in the sample, and was successfully circumvented. Modifying the LC mobile phase with $\mathrm{HCOONH}_{4}(0.2$ $\mathrm{mM}$ ) increased both ESI efficiency and capacity. This led to significantly enhanced analytical performance of our newly developed method, i.e., improved sensitivity, reduced matrix effects, and enlarged linear dynamic ranges compared with previously reported methods. Due to these advancements, our new procedure requires a 5-min chromatographic run time and uses 20- $\mu$ L plasma samples. Collectively, our new analytical method should facilitate PK measurements of various ginkgo products.

\section{Acknowledgments}

The authors acknowledge support for this work by the Chinese Ministry of Science and Technology (2005CB523403), the National Natural Science Foundation of China (30772772), the Science and Technology Commission of Shanghai Municipality (07DZ19703), and SIMM-CAS (07G604J049).

\section{References}

1. Oken, B. S.; Storzbach, D. M.; Kaye, J. A. The Efficacy of Ginkgo biloba on Cognitive Function in Alzheimer Disease. Arch. Neurol. 1998, 55, 1409 1415.

2. Mauri, P.; Simonetti, P.; Gardana, C.; Minoggio, M.; Morazzoni, P.; Bombardelli, E.; Pietta, P. Liquid Chromatography/Atmospheric Pressure Chemical Ionization Mass Spectrometry of Terpene Lactones in Plasma of Volunteers Dosed with Ginkgo biloba L. Extract. Rapid Commun. Mass Spectrom. 2001, 15, 929-934.

3. Mauri, P.; De Palma, A.; Pozzi, F.; Basilico, F.; Riva, A.; Morazzoni, P.; Bombardelli, E.; Rossoni, G. LC-MS Characterization of Terpene Lactones in Plasma of Experimental Animals Treated with Ginkgo biloba Extracts Correlation with Pharmacological Activity. J. Pharm. Biomed. Anal. 2006, 40, 763-768.

4. Zhao, Y.; Wang, L.; Bao, Y.-W.; Li, C. A Sensitive Method for the Detection and Quantification of Ginkgo Flavonols from Plasma. Rapid Commun. Mass Spectrom. 2007, 21, 971-981.

5. Ding, S.-J.; Dudley E.; Chen L.-J., Plummer, S.; Tang, J.-D.; Newton, R. P.; Brenton, A. G. Determination of Active Components of Ginkgo biloba in Human Urine by Capillary High-Performance Liquid Chromatography/ Mass Spectrometry with On-Line Column-Switching Purification. Rapid Commun. Mass Spectrom. 2006, 20, 3619-3624.

6. Li, L.; Liang, S.-P.; Du, F.-F.; Li, C. Simultaneous Quantification of Multiple Licorice Flavonoids in Rat Plasma. J. Am. Soc. Mass Spectrom. 2007, 18, 778-782.

7. Wang, L.; Sun, Y.; Du, F.-F.; Niu, W.; Lu, T.; Kan, J.-M.; Xu, F.; Yuan, K.-H.; Qin, T.; Liu, C.-X., Li, C. LC-electrolyte Effects Improve the Bioanalytical Performance of Liquid Chromatography/Tandem Mass Spectrometric Assays in Supporting Pharmacokinetic Study for Drug Discovery. Rapid Commun. Mass Spectrom. 2007, 21, 2573-2584.

8. Johnson, E. L.; Reynolds, D. L.; Wright, D. S.; Pachla, L. A. Biological Sample Preparation and Data Reduction Concepts in Pharmaceutical Analysis. J. Chromatogr. Sci. 1988, 26, 372-379.

9. Cech, N. B.; Enke, C. G. Practical Implications of Some Recent Studies in Electrospray Ionization Fundamentals. Mass Spectrom. Rev. 2001, 20, 362-387.

10. Fernandez de la Mora, J.; Loscertales, I. G. The Current Emitted by Highly Conducting Taylor Cones. J. Fluid Mech. 1994, 260, 155-184. 